Voix et Images

volxetimages

\title{
De Dubé à la Marmaille : autopsie d'une production théâtrale
}

\section{Bernard Andrès}

Volume 3, numéro 2, décembre 1977

Victor-Lévy Beaulieu

URI : https://id.erudit.org/iderudit/200113ar

DOI : https://doi.org/10.7202/200113ar

Aller au sommaire du numéro

\section{Éditeur(s)}

Les Presses de l'Université du Québec

\section{ISSN}

0318-9201 (imprimé)

1705-933X (numérique)

Découvrir la revue

\section{Citer cet article}

Andrès, B. (1977). De Dubé à la Marmaille : autopsie d'une production théâtrale. Voix et Images, 3(2), 330-334. https://doi.org/10.7202/200113ar d'utilisation que vous pouvez consulter en ligne.

https://apropos.erudit.org/fr/usagers/politique-dutilisation/ 


\section{De Dubé à la Marmaille: autopsie d'une production théâtrale}

Pour le lancement du Théâtre Denise-Pelletier où s'est installé en octobre dernier la Nouvelle Compagnie théâtrale, on a présenté en alternance, Zone de Dubé et la Vie à trois étages, création collective de la Marmaille. Deux spectacles, deux conceptions du théâtre avec de l'un à l'autre, comme en abrégé, l'évolution du théâtre québécois de 1953 à nos jours. A vingt-cinq ans de distance, des jeunes dans la vingtaine se risquent dans l'entreprise théâtrale. Dubé et ses amis de la Jeune Scène, la Marmaille et Marie-Francine Hébert, Claire Leroux, Claude Roussin et Michel Garneau. D'une part un auteur et des comédiens; de l'autre une troupe et ses auteurs: première différence, de taille. L'évolution se marque dans le rapport auteur/comédien, selon l'importance que prend (ou perd) progressivement l'un ou l'autre élément.

Dubé travaille des textes qu'il confie à des comédiens en suivant sa propre "évolution", d'un réalisme à contenu social portant sur les milieux défavorisés, jusqu'à un réalisme psychologique cantonné à la petite-bourgeoisie québécoise. A l'image de nombreuses jeunes troupes actuelles, la Marmaille suit la démarche inverse. C'est elle en tant que troupe qui recherche un auteur pour finaliser un travail amorcé en atelier d'écriture avec le public (enfants ou adultes de divers milieux sociaux - surtout défavorisés). Réalistes à l'occasion, ces productions sont plutôt fantaisistes 
et expérimentales: il s'agit de voir comment la création se manifeste selon le milieu social et quel type de pièce est le plus efficace pour le public (théâtre d'auteur ou création «collective $»)^{1}$.

On pourrait ainsi schématiser la démarche de Dubé: l'auteur de Zone et du Réformiste a trouvé son public en s'éloignant du réalisme populaire de ses débuts ${ }^{2}$. II s'adresse désormais à une petite-bourgeoisie dont il joue avec les contradictions plus qu'il ne les dénonce, résolvant plus volontiers la crise au niveau psychologique et individuel, qu'au niveau collectif. La dialectique du Réformiste (1977) est assez significative, qui ne représente des conflits sociaux que pour mieux illustrer un conflit de générations, figurer une mésentente matrimoniale, concrétiser un dilemme philosophique ou un désarroi psychologique. Au centre, l'Homme («l'homo burgensis $»$ ), autour: sa femme, son fils, sa maîtresse, ses idéaux, son école. Plus loin, vaguement: des agiteurs syndicalistes sans scrupule, dans une société en décomposition. Loin de se dérouler, l'«action" s'enroule dans un mouvement centripète autour de cet homme, l'enferme dans son cocon idéologique, jusqu'à l'isolement final. II en est de même du rapport aux artisans de la scène: apparemment ce théâtre est fait "pour » eux, dans la mesure où l'on a précisément des Rôles et des Fonctions asur mesure". Mais à y bien penser, la même force centripète ramène tout au texte. Dans l'optique traditionnelle du theâtre littéraire, tout et tous concourent à servir le texte, à l'étayer, à l'élever sur les planches: Dans son étude sur Dubé, Jocelyne Mathé rappelle bien ce que doit l'auteur aux artisans de son théâtre: "le travail des metteurs en scène, tels que Louis-Georges Carrier, Jean-Paul Fugère, Jean Gascon, Paul Blouin, apportent aux pièces présentées des perfections techniques que l'auteur sait apprécier. Et l'émotion tragique est souvent amplifiée par les trouvailles des comédiens. Maximilien Laroche raconte, entre autres, un jeu de scène de Gilles Pelletier "qui en une simple attitude fixe aux yeux des spectateurs l'état d'âme du personnagen. Dans Un simple soldat, l'acteur qui jouait le rôle de Joseph, a eu l'idée de prendre la position du fœtus devant la porte de chambre de son père, illustrant ainsi jusqu'à quel point le soldat est demeuré l'enfant marqué par la mort de sa mère et le remariage de son père. Et Marcel Dubé rend lui-même hommage à Monique Miller: “... C'est elle aussi, je pense, qui en partie m'a fait comprendre ce qu'était véritablement le sens du tragique au théâtre." On sait enfin que Dubé a créé des rôles pour ses plus fidèles interprètes tels que Jean Duceppe et Andrée Lachapelle ${ }^{3}$.

A cette conception centripète du texte théâtral qui reproduit indéfiniment les valeurs humanistes, répond un langage théâtral qui les conteste et se remet lui-même en question à chaque phrase de sa production: de la conception à la réalisation du spectacle. C'est dans cette optique que travaillent au Québec des troupes comme la Marmaille, qui n'emploient les auteurs qu'à l'occasion, pour finaliser telle ou telle étape... ou qui s'en passent en se substituant à lui. La genèse de la Vie à trois étages, de la Marmaille, mérite qu'on s'y arrête 4 . 
Disposant d'un budget d'atelier (payé par la NCT), les comédiens s'emploient de janvier à avril 77 à rencontrer leur public spécifique: les gens du quartier Hochelaga-Maisonneuve (troisième âge dans les résidences, adultes au CLSC, dans les brasseries, au Centre culturel de l'Est et chez eux, adolescents dans les institutions scolaires, etc.). Entrevues et sélection d'une soixantaine de personnes, aboutissant à un tableau d'ensemble sur l'histoire du quartier et ses préoccupations immédiates. Une vingtaine d'ateliers d'écriture groupant chacun trois de ces personnes, trois comédiens et un animateur, arrêtent un certain nombre de thèmes. Les comédiens entreprennent alors d'explorer ces champs en improvisant sur ce matériel, pour aboutir à un canevas soumis à la NCT. Avant les trois mois de pause où ils laissent décanter leur projet, le choix idéologique est arrêté: il ne s'agit pas d'enregistrer et de transcrire, de reproduire le réel, mais d'y apporter une dimension critique à partir des virtualités de changement exprimées plus ou moins confusément par ce groupe social (qui sera aussi leur public). La création collective du spectacle proprement dit intervient à la rentée: deux mois de répétitions où le canevas est modifié en vue d'un équilibre entre les éléments réalistes et les séquences distanciées. Parler le langage du télé-feuilleton pour atteindre ce public, mais subvertir ce discours pour éviter l'identification stérile au personnage (les comédiens portent des masques). Insister sur des situations plutôt que sur des personnages. Rester critique face au quotidien. Transposer le réel par des éléments fantaisistes et distanciés pour éviter ce «naturalisme québécois» qui guette aussi bien Dubé que Tremblay.

On aboutit à cette production que certains auront vue. La Vie à trois étages, c'est d'abord un volume scénique occupé par trois appartements superposés: la famille des proprios, le jeune couple du premier et "la gang" du troisième. Les éléments réalistes (conflits de génération, de famille, de couple, de communauté) se trouvent transcendés par un projet commun: résister à l'expropriation. Ancrage de cette intrigue dans I'historique du quartier (du Marché Maisonneuve au Stade Olympique en passant par le Château Dufresne, tout un passé témoigne des transformations subies par le quartier). Intégration de la musique, des chansons et du mime dans le langage scénique, et le tour est joué: entendons le processus cyclique d'une création qui passe par le public de la conception à la réalisation (et qui ne s'interdit pas de modifier le résultat en fonction des réactions - souhaitées - du dit public).

Une telle démarche repose évidemment sur le critère d'efficacité immédiate de la pièce et relègue au dernier plan l'idée d'un théâtre pour la postérité. Mais qui décide de la postérité d'une œuvre? Dans dix ans, vingt ans, on parlera encore de Zone... peut-être, plus tellement (ou du tout) de la Vie à trois étages. Et alors? On n'en parlera plus, surtout si Hochelaga-Maisonneuve n'existe plus. Mais si ce quartier subsiste (en tant que milieu humain), c'est que la NCT y sera toujours. Peut-être grâce 
à des œuvres qui l'auront connue, cette postérité (mais j'en doute). Surtout grâce à beaucoup d'autres spectacles comme la Vie qui redonnent goût à la collectivité de se reprendre en main et de sauver son milieu, "son héritage, son langage". Eux seuls pratiquent l'ancrage humain et culturel indispensable à la survie d'une institution théâtrale transplantée comme cette compagnie. Ainsi décentrée dans l'Est, la NCT n'élargira peutêtre pas son public actuel mais elle en atteindra sûrement un autre qui sans elle, n'aurait pas eu accès au théâtre, et qui la fera vivre et qu'elle fera vivre. Il suffisait de voir les gens du quartier, ravis, fouiner dans "leur" (ex) cinéma Granada une semaine avant la Première, pour réaliser qu'ils étaient bien chez eux... et qu'il fallait tout mettre en œuvre pour qu'ils y demeurent. Pourtant “la direction a changé»: les directeurs mais aussi le sens de l'entreprise. Malgré tout, ils flânaient comme chez eux, interpelant le contremaître, donnant leur avis sur la décoration et félicitant cavalièrement la nouvelle direction en prenant à parti sans le savoir... Françoise Gratton qui me faisait visiter l'édifice! Quant à cette postérité qu'on attribue aisément au bon goût et aux "valeurs éternelles " véhiculées par l'œuvre, on oublie qu'elle répond avant tout aux besoins culturels (et économiques) d'un milieu à une époque donnée. Longtemps déterminée par les impératifs et intérêts de l'élite ou de "l'homo burgensis " évoqué plus haut, la postérité risque d'en surprendre plus d'un, parmi ceux qui serraient la culture dans les rayons de leur bibliothèque. On n'enferme pas le théâtre, tout au plus en laisse-t-on une trace dérisoire dans le texte dramatique.

Certes, le texte de cette pièce ne sera pas "littéraire", au sens ou les mots n'auront plus le grand rôle dans la partition théâtrale. Ils auront simplement l'emploi que le synopsis cinématographique réserve au dialogue dans la bande sonore, parallèlement à tous les autres codes sémiologiques investis (musical, kinésique, expressif, symbolique, etc.). Seule une telle mise en partition du phénomène théâtral permettra d'apprécier à sa juste mesure le texte, non pas en soi, mais par rapport aux autres systèmes de signes qu'il esquisse, explicite ou redouble. Même si leurs "textes"semblent moins élaborés que ceux de leurs aînés, les jeunes comédiens (et la plupart des jeunes dramaturges) sont infiniment plus conscients, pour leur part, de la spécificité du discours théâtral et de sa fonction sociale en milieu québécois.

Bernard Andrès

1. Sur les différentes formes que peuvent revêtir ces types de productions voir, dans Jeu, $n^{\circ}$ 4: "Aspects de la création collective québécoise", de Fernand Villemure et aAuteur/création collective: mythe et réalité", de Michel Vaïs.

2. Le caractère schématique de ces remarques s'explique par les limites mêmes de cette chronique. Une étude d'ensemble reste à faire autour de cette remarque de Laurent Mailhot: "Au moment où il atteignait à la dimension poli- 
tique du théâtre, Dubé s'en détourne pour s'attacher aux tares et traumatismes personnels. o (In la Littérature québécoise, Paris, PUF, 1974, p. 109.)

3. Jocelyne Mathé, "Dubé", dans le Théatre canadien-français, "Archives des lettres canadiennesn, tome V, Montréal, Fides, 1976, p. 510-511.

4. Je reprends ici la démarche exposée par la Marmaille dans le communiqué du 3 octobre 1977 (dossier de presse de la NCT), ainsi que les grandes lignes d'une entrevue que m'accordaient le 11 octobre 1977 Francine Merville, Daniel Meilleur et Gilbert David. 